\title{
Locus de controlo e adesão terapêutica em adultos emergentes e adultos com condições crónicas de saúde: 0 papel moderador do grupo etário
}

\author{
Jéssica Reis ${ }^{1}$, Raquel Pires ${ }^{1,2}$ \& Neuza Silva ${ }^{1,3}$ \\ ${ }^{1}$ EPCV, Universidade Lusófona de Humanidades e Tecnologias \\ ${ }^{2}$ Associação para Investigação e Desenvolvimento da Faculdade de Medicina, Centro Cardiovascular da \\ Universidade de Lisboa \\ ${ }^{3}$ Centro de Investigação do Núcleo de Estudos e Intervenção Cognitivo-Comportamental, FPCE da \\ Universidade de Coimbra
}

\begin{abstract}
Resumo: Este estudo pretendeu analisar as associações entre locus de controlo (LC) e adesão terapêutica em indivíduos com condições crónicas de saúde, assim como o papel moderador do grupo etário nestas relações. A amostra incluiu 61 adultos emergentes (18-25 anos) e 60 adultos (26-64 anos) com doença crónica e a receberem tratamentos. Os participantes preencheram um questionário de dados clínicos/sociodemográficos e instrumentos de avaliação do LC e da adesão terapêutica. Não foram encontradas diferenças significativas no LC nem na adesão terapêutica entre grupos etários. Uma maior tendência para um LC externo-acaso associou-se a menores níveis de adesão terapêutica, independentemente do grupo etário, enquanto a associação negativa entre LC interno e adesão e a associação positiva entre LC externo-outros poderosos e adesão foram significativas apenas para os adultos emergentes. Estes resultados sugerem que o LC pode influenciar a adesão aos tratamentos, pelo que deve ser considerado como alvo terapêutico nas intervenções psicossociais.
\end{abstract}

Palavras-chave: Adesão terapêutica; Adultos; Condições crónicas de saúde; Adultos emergentes; Locus de controlo da saúde.

\begin{abstract}
Locus of control and adherence to treatments in adults and emerging adults with chronic health conditions: The moderating role of age-group: This study was aimed at examining the associations between locus of control (LC) and adherence to treatments in individuals with chronic health conditions, as well as the moderating role of age-group on these associations. The sample included 61 emerging adults (18-25 years) and 60 adults (26-64 years) with a chronic condition and who were receiving treatments. Participants completed a clinical/sociodemographic datasheet and self-report questionnaires assessing LC and adherence to treatments. No significant differences were found in LC orientation or adherence to treatments between age-groups. A greater likelihood of having an external LC by chance was associated with lower adherence independently of age-group, while the negative link between internal LC and adherence and the positive link between external LC by powerful others and adherence were statistically significant only for emerging adults. These results suggest that LC may influence adherence to treatments and, therefore, should be considered as an intervention target in psychosocial interventions.
\end{abstract}

Keywords: Adherence to treatments; Adults; Chronic health conditions; Emerging adults; Health locus of control.

As condições crónicas de saúde constituem um problema de saúde pública, sendo as principais causas de mortalidade e morbilidade observadas nos países desenvolvidos e em vias de desenvolvimento, originando uma enorme sobrecarga ao nível dos cuidados de saúde (Sousa, Landeiro, Pires, \& Santos, 2011). As pessoas com uma doença crónica confrontam-se com diversas circunstâncias stressantes que advêm da diminuição da independência, evolução da doença e, em alguns casos, da necessidade de mudança de comportamento para dar resposta às prescrições terapêuticas (Wiebe \& Christensen, 1996). Neste sentido, é imperativo que o doente seja capaz de integrar esse acontecimento não-normativo na sua vida, procurando um equilíbrio entre as suas atividades cotidianas e a gestão da doença e dos tratamentos (Sousa et al., 2011). A concomitância entre as exigências da doença e as tarefas

${ }^{1}$ Endereço para correspondência: Centro de Investigação do Núcleo de Estudos e Intervenção Cognitivo-Comportamental, Faculdade de Psicologia e de Ciências da Educação da Universidade de Coimbra, Rua do Colégio Novo, 3000-115 Coimbra, Portugal. E-mail: neuzambsilva@gmail.com 
desenvolvimentais normativas do período de transição para a idade adulta (e.g., adoção de novos papéis emocionais e sociais, independência da família) pode comprometer o processo de adaptação e a autonomia na gestão dos cuidados de saúde (Kyngäs, Kroll, \& Duffy, 2000). No entanto, os resultados de adaptação psicossocial não dependem diretamente das características clínicas da condição de saúde e dos tratamentos, mas de complexas interações entre a perceção de stresse decorrente da doença e dos recursos disponíveis para enfrentar a situação (Lazarus \& Folkman, 1984). O estudo das associações entre locus de controlo e adesão terapêutica em adultos emergentes, em comparação com adultos, poderá contribuir para uma melhor compreensão dos processos de adaptação e para a identificação de potenciais alvos terapêuticos em intervenções psicossociais com vista à promoção da saúde e à diminuição da morbilidade decorrente da condição crónica de saúde (Blount et al., 2007).

\section{Adultez Emergente: Desenvolvimento e Saúde}

Tem-se observado um aumento do número de adolescentes e jovens adultos com condições crónicas de saúde (Sawyer, Drew, Yeo, \& Britto, 2007), estimando-se uma prevalência de cerca de 10\% nestas faixas etárias (Suris, Michaud, \& Viner, 2004). Enquanto a medicina e a psicologia da saúde pediátrica se têm focado na saúde e adaptação psicossocial de crianças e adolescentes até aos 18 anos de idade, os estudos com adultos tendem a desconsiderar o período entre a adolescência tardia e o início da idade adulta como um período desenvolvimental distinto (Arnett, 2000). Como resultado, a investigação empírica sobre a adaptação a uma condição crónica de saúde neste período de transição é praticamente inexistente (Anderson \& Wolpert, 2004).

Fundamentado pelas alterações nos padrões sociais e culturais dos países industrializados (e.g., prolongamento da escolaridade; adiamento do casamento e parentalidade), Arnett (2000) propôs a designação de "adultos emergentes" para distinguir a fase do ciclo de vida dos indivíduos entre os 18 e os 25 anos. Esta "nova" fase desenvolvimental é caracterizada pela mudança e exploração dos possíveis percursos de vida, e distingue-se teórica e empiricamente da adolescência e da idade adulta jovem ao nível demográfico, identitário e subjetivo (Arnett, 2000, 2006; Andrade, 2010). Enquanto a maioria dos adolescentes até aos 18 anos vive com os seus pais, está a lidar com as mudanças físicas da puberdade, frequenta a escola secundária e faz parte de uma cultura de pares sediada na escola, a partir dos 18 anos a maioria dos jovens já terminou a escola secundária, deixou a casa dos pais e atingiu a maioridade legal numa série de aspetos. Adicionalmente, e ao contrário dos adultos jovens que já são física, emocional e financeiramente independentes da família de origem, já estabeleceram a sua identidade social e relações de intimidade duradouras, e estão focados na prossecução de objetivos profissionais (Erickson, 1980), os adultos emergentes estão ainda em processo de obtenção da educação e formação necessárias para uma ocupação a longo prazo e de formação da sua identidade a partir da exploração das possibilidades de percursos relacionais, laborais e de visão do mundo, ainda não são casados e não têm filhos, e partilham a perceção subjetiva de ainda não terem atingido completamente a idade adulta no sentido de aceitarem a responsabilidade por si mesmos e tomar decisões independentes (Arnett, 2000). Mendonça, Andrade e Fontaine (2009), no seu estudo sobre os marcadores de entrada na idade adulta, confirmam que estas especificidades demográficas, identitárias e subjetivas se verificam também no contexto sociocultural português.

Nesta fase, é então esperado que o jovem aprenda a lidar com os novos papéis emocionais e sociais e adote comportamentos responsáveis, incluindo o autocuidado e a gestão da sua saúde (Taddeo, Egedy, \& Frappier, 2008). Estudos prévios têm mostrado que atributos psicossociais que se desenvolvem durante a adultez emergente, como a identidade (e.g., incorporação de características de estilos de vida saudáveis no conceito de si próprio) e auto-eficácia, são indicadores importantes dos comportamentos de saúde (Nelson, Story, Larson, Neumark-Sztainer, \& Lytle, 2008; Storer, Cychosz, \& Anderson, 1997). Sendo esta uma fase em que os padrões de comportamento e de autocuidado são explorados, as intervenções psicoeducativas podem ter um impacto crítico sobre a saúde a longo prazo (Kelly, Kratz, Bielski, \& Rinehart, 2002).

\section{Condições Crónicas de Saúde e Adesão Terapêutica}

A adesão terapêutica, definida como "the extend to which the time history of drug administration corresponds to the prescribed regímen" (Molassiotis et al., 2002, p. 301), é considerada um determinante fundamental do êxito do tratamento (Linhares et al., 2014). Nos doentes crónicos em geral, a taxa de adesão terapêutica ronda os 50\% (Molassiotis et al., 2002), representando assim uma das maiores preocupações ao nível da saúde pública (Kurita \& Pimenta, 2004). Os adolescentes e adultos emergentes apresentam taxas de não-adesão superiores àquelas observadas noutras faixas etárias (Bleyer, 2002; Griva, Myers, \& Newman, 2000; Sirey et al., 2001), com valores que variam entre os $27 \%$ e os $60 \%$ (Kondryn, Edmondson, Hill, \& Eden, 2011; Suris et al., 2004), o que pode contribuir para o agravamento 
da doença, sobrecarga dos serviços de saúde e decréscimo da sua qualidade de vida (Taddeo et al., 2008; Trevino, Fasciano, \& Prigerson, 2013). Determinar as taxas de adesão de forma precisa é uma tarefa complexa, uma vez que estas podem depender do tipo de doença, da complexidade do regime terapêutico (Bond \& Hussar, 1991, cit. in Delgado \& Lima, 2001) e do nível socioeconómico dos indivíduos (Reiners, Azevedo, Vieira, \& Arruda, 2008).

As baixas taxas de adesão terapêutica na fase de transição para a idade adulta podem ser justificadas pelo pensamento abstrato pouco desenvolvido nesta idade, autocentração, instabilidade relacional, laboral e na visão do mundo, e pela imaturidade na previsão de consequências e de vulnerabilidade (Hinds et al., 2000; Suris et al., 2004; Taddeo et al., 2008). As condições crónicas de saúde são caracterizadas pelo seu curso imprevisível, alterações nas capacidades, aparência e perspetivas futuras e pela dependência prolongada dos serviços de saúde. Neste sentido, viver com uma doença crónica requer que os indivíduos estejam em contínua adaptação devido às mudanças permanentes no estilo de vida. Os adolescentes e adultos emergentes com estas condições de saúde apresentam necessidades adicionais e prioridades pessoais que diferem dos seus pares saudáveis, nomeadamente limitações nas atividades e restrições na vida diária e social (Blum et al., 1993), pois os regimes terapêuticos geralmente acarretam implicações negativas nas suas relações, levando à dependência social e ao isolamento temporário (Kyngäs et al., 2000).

\section{Locus de Controlo da Saúde e Adesão Terapêutica}

O diagnóstico de uma condição crónica de saúde pode ser encarado como uma situação potencialmente indutora de stresse, não só pelo impacto imediato do diagnóstico, mas também pelos desafios diários subsequentes (Röder \& Boekaerts, 1999). De acordo com o modelo de Lazarus e Folkman (1984), o significado de um evento é determinado pelos processos de avaliação cognitiva, que se dividem na avaliação primária, na qual a pessoa avalia o significado individual ou a relevância do acontecimento (e.g., acontecimento irrelevante, benéfico ou ameaçador), e na avaliação secundária, que consiste na avaliação dos recursos pessoais de coping disponíveis para lidar com as exigências da situação (Samson \& Siam, 2008), nomeadamente a perceção de controlo sobre o acontecimento. 0 stresse ocorre quando as exigências da situação excedem os recursos e capacidades do indivíduo para a enfrentar (Folkman, 1984). No contexto específico da doença crónica, resultados mais ou menos adaptativos serão então determinados, não só pelas características clínicas, mas também pela perceção individual de stresse decorrente da doença e/ou dos tratamentos (Hesselink et al., 2004; Röder \& Boekaerts, 1999).

O locus de controlo surge neste modelo como a perceção de controlo que os indivíduos detêm sobre os outcomes importantes, sendo que estas crenças de controlo podem influenciar a avaliação cognitiva primária e secundária (Folkman, 1984) e predizer a adesão terapêutica. 0 conceito de locus de controlo deriva da teoria da aprendizagem social (Rotter, 1966), consistindo na perceção desenvolvida pelas pessoas acerca da origem e controlo das situações que experienciam (Barletta, 2010; Carrijo \& Coleta, 2007). Aplicado ao contexto da saúde, o locus de controlo refere-se à crença que os indivíduos têm sobre quem ou qual o agente que determina o seu estado de saúde (Wallston, 1982) e reflete as expectativas individuais sobre os resultados de saúde (Christensen, Wiebe, Benotsch, \& Lawton, 1996). Neste sentido, os indivíduos que creem que a doença surgiu como resultado das suas ações e que acreditam que os resultados de saúde são controláveis por si, detêm um locus de controlo interno (Barletta, 2010; Kurita \& Pimenta, 2004). Já os indivíduos que tendem a fazer atribuições externas e que acreditam que a sua saúde depende da ação de outros, como os profissionais de saúde, ou que os seus resultados de saúde são definidos casualmente, apresentam um locus de controlo externo (Barletta, 2010; Berglund, Lytsy, \& Westerling, 2014; Carrijo \& Coleta, 2007).

A variação do locus de controlo consoante a faixa etária é discutida na literatura de forma controversa. Alguns autores reportam que, à medida que a idade avança, os indivíduos tendem a percecionar menor controlo pessoal sobre as situações, o que pode ser justificado pela maior dificuldade dos indivíduos mais velhos em avaliar os seus acontecimentos stressores como modificáveis, visto que muitas das mudanças que acompanham o envelhecimento não são controláveis, e pela maior importância que atribuem às fontes externas de controlo (Aldwin, 1991; Lachman, 1986; Lachman, Neupert, \& Agrigoroaei, 2011). Lachman (1986) acrescenta que, embora seja dado maior reconhecimento a fontes externas de controlo, os adultos continuam a manter, ao longo dos anos, um sentido de internalidade. No entanto, outros estudos não encontraram diferenças significativas em função da idade (Blanchard-Fields \& Robinson, 1987), ou relatam que as perceções de controlo interno tendem a acentuar-se com a idade, principalmente a partir dos 30-39 anos de idade, mantendo-se relativamente estáveis durante toda a vida adulta e sofrendo apenas um decréscimo não significativo a partir dos 60 anos (Lao, 1974). Estes autores argumentam que as experiências de vida, e consequente aumento do domínio e competência do indivíduo ao longo da sua maturação, vão conduzir a um maior senso de controlo. 
Apesar da inexistência de estudos focados no locus de controlo dos adultos emergentes, a autocentração e progressiva independência e perceção de autocontrolo que caracterizam este grupo desenvolvimental são partilhadas pelos adolescentes (Arnett, 2000; Taddeo et al., 2008). No entanto, o diagnóstico de uma condição crónica de saúde pode induzir sentimentos de impotência e ineficácia pessoal (Taddeo et al., 2008). Estudos empíricos com adolescentes concluíram que o diagnóstico de determinadas doenças crónicas está associado à redução da perceção de controlo sobre o futuro da saúde (Kellerman, Zelter, Ellenberg, Dash, \& Rigler, 1980) e que um diagnóstico de cancro na adolescência está associado à perda de controlo, na qual surgem sentimentos de desamparo, comprometendo o desenvolvimento do sentido de autonomia e da independência (Jamison, Lewis, \& Burish, 1986).

Apesar dos doentes crónicos dependerem dos profissionais de saúde para o aconselhamento e intervenção, Wallston (1992) concluiu que os comportamentos de adesão se encontram mais associados a expectativas de competência sobre os resultados de saúde. Vários estudos empíricos reportam associações significativas entre a tendência para um locus de controlo predominantemente interno e uma maior adesão terapêutica (e.g., Katz, Yelin, Eisner, \& Blanc, 2002; Molassiotis et al., 2002; Rybarczyk, DeMarco, DeLaCruz, Lapidos, \& Fortner, 2001; Tillotson \& Smith, 1996), e entre uma orientação de locus de controlo externo, fundamentado na confiança nos profissionais de saúde, e uma maior adesão terapêutica, em indivíduos com condições crónicas de saúde (Christensen et al., 1996). No entanto, outros estudos não encontraram associações significativas entre o locus de controlo e comportamentos de adesão aos tratamentos (Carrijo \& Coleta, 2007), sugerindo que outros fatores, nomeadamente a etapa desenvolvimental possa interferir de forma significativa nesta relação.

\section{O Presente Estudo: Objetivos e Hipóteses}

Apesar da grande controvérsia acerca das idades que delimitam a fase de transição para a idade adulta, justificada pelas mudanças sociais e económicas nas sociedades industrializadas e pela heterogeneidade de percursos que caracteriza esta fase (Dellamann-Jenkins, Blankemeyer, \& Pinkard, 2001), neste estudo foi adotada a perspetiva de Arnett (2000), que classifica esta fase entre os 18 e os 25 anos. Embora a faixa etária dos adultos compreenda indivíduos em diferentes fases do ciclo da vida (jovens adultos e adultos de meia-idade), a sua categorização num só grupo justifica-se pelas características desenvolvimentais que apresentam em comum, como a maturação física, sexual e cognitiva plena, a conquista da independência, e a estabilidade relacional, laboral e da sua visão do mundo (Arnett, 2000; Fleming, 2008).

No contexto da adaptação psicossocial às condições crónicas de saúde, o locus de controlo da saúde foi considerado neste estudo como um indicador da avaliação cognitiva secundária da condição crónica de saúde enquanto acontecimento potencialmente indutor de stresse, e a adesão terapêutica foi analisada enquanto resultado adaptativo. Com base no modelo de stresse e coping (Lazarus \& Folkman, 1984), este estudo pretendeu: (1) comparar o locus de controlo da saúde e a adesão terapêutica entre adultos emergentes (18-25 anos) e adultos jovens ou de meia-idade (26-64 anos); (2) analisar as associações entre locus de controlo interno/externo e adesão terapêutica; e (3) analisar o papel moderador do grupo etário na relação entre locus de controlo interno/externo e adesão terapêutica.

Fundamentadas pela revisão de literatura, foram delineadas as seguintes hipóteses: (H1) espera-se uma maior adesão terapêutica nos adultos, em comparação com os adultos emergentes; (H2) espera-se que uma maior tendência para um locus de controlo interno ou externo fundamentado na crença de que a saúde depende da ação de outros poderosos (e.g., profissionais de saúde) esteja associada a maior adesão terapêutica; (H3) espera-se que um locus de controlo predominantemente externo fundamentado na crença de que a saúde depende do acaso esteja associado a menor adesão terapêutica. Em relação às diferenças na orientação predominante do locus de controlo da saúde entre os adultos emergentes e os adultos com condições crónicas de saúde, não foi possível elaborar quaisquer hipóteses devido, por um lado, à inexistência de literatura focada na adultez emergente, e, por outro lado, aos resultados divergentes encontrados nos estudos com adultos. Devido à natureza exploratória das análises do papel moderador do grupo etário, também não foram elaboradas hipóteses a este respeito.

\section{MÉTODO}

\section{Participantes e Procedimentos}

Este estudo integrou um projeto alargado intitulado Processos e Resultados de Adaptação Psicossocial em Indivíduos Saudáveis e com Condições Crónicas de Saúde, aprovado pela Comissão de Ética e Deontologia para a Investigação Científica (CEDIC) da Universidade Lusófona de Humanidades e Tecnologias. Este projeto teve um desenho quantitativo transversal, de natureza correlacional. Os participantes para este estudo foram selecionados através do método de amostragem não probabilística por conveniência, entre Março e Maio de 2016, de acordo com os seguintes critérios de inclusão: (1) idade compreendida entre 18 
e 64 anos; (2) diagnóstico clínico de uma condição crónica de saúde, de acordo com a CID-10 (WHO, 2010); (3) estarem atualmente a receber tratamentos para a sua condição de saúde; (4) não terem diagnóstico e não estarem a fazer tratamentos para perturbação psicológica/psiquiátrica; e (5) missingvalues numa percentagem inferior a $20 \%$.

Os dados foram recolhidos por meio de questionários de autorresposta, utilizando dois métodos distintos: recolha de questionários em formato de "papel e lápis" em contexto comunitário e através de uma plataforma online divulgada pelas redes sociais e pelos contatos dos investigadores. Em ambos os métodos de recolha de dados, foi facultada aos participantes informação detalhada sobre os objetivos e procedimentos do estudo e solicitado consentimento informado. Foi garantido o anonimato dos participantes e das respostas, em cumprimento com os princípios éticos relativos à investigação com participantes humanos (APA, 2010) e com a Declaração de Helsínquia (WMA, 2008).

As características sociodemográficas e clínicas dos participantes (61 adultos emergentes e 60 adultos) são apresentadas na Tabela 1 .

Tabela 1. Características sociodemográficas e clínicas da amostra.

\begin{tabular}{|c|c|c|c|}
\hline & $\begin{array}{l}\text { Adultos emergentes } \\
(n=61)\end{array}$ & $\begin{array}{l}\text { Adultos } \\
(n=60)\end{array}$ & $\begin{array}{c}\begin{array}{c}\text { Diferenças entre } \\
\text { grupos }\end{array} \\
\end{array}$ \\
\hline \multicolumn{4}{|l|}{ Características sociodemográficas } \\
\hline Idade, $M(D P)$ & $22.38(2.14)$ & $37.93(8.75)$ & - \\
\hline Sexo, $n(\%)$ & & & $\chi_{(1)}^{2}=0.89$ \\
\hline Feminino & $20(32.8 \%)$ & $15(25.0 \%)$ & \\
\hline Masculino & $41(67.2 \%)$ & $45(75.0 \%)$ & \\
\hline Escolaridade em anos, $M(D P)$ & $13.65(2.46)$ & $14.30(3.94)$ & $t_{(118)}=-1.08$ \\
\hline Situação profissional, $n(\%)$ & & & $\chi_{(3)}^{2}=33.08^{* *}$ \\
\hline Estudante & $28(45.9 \%)$ & $5(8.3 \%)$ & \\
\hline Estudante-trabalhador(a) & $15(24.6 \%)$ & $7(11.7 \%)$ & \\
\hline Trabalhador(a) & $13(21.3 \%)$ & $34(56.7 \%)$ & \\
\hline Desempregado(a)/ Reformado(a) & $4(6.6 \%)$ & $13(21.7 \%)$ & \\
\hline Estado civil, $n(\%)$ & & & $\chi^{2}{ }_{(2)}=47.37^{* *}$ \\
\hline Solteiro(a) & $57(93.4 \%)$ & $20(33.3 \%)$ & \\
\hline Casado(a)/união de facto & $4(6.6 \%)$ & $36(60.0 \%)$ & \\
\hline Divorciado(a)/ Separado(a)/ Viúvo(a) & $0(0.0 \%)$ & $4(6.7 \%)$ & \\
\hline Elementos do agregado familiar, $M(D P)$ & $3.15(1.18)$ & $2.80(1.12)$ & $t_{(119)}=1.66$ \\
\hline Rendimento do agregado familiar, $n(\%)$ & & & $\chi^{2}{ }_{(2)}=1.26$ \\
\hline Menos de $583 € /$ mês & $9(14.8 \%)$ & $5(8.3 \%)$ & \\
\hline Entre 583 e 1666 €/mês & $35(57.4 \%)$ & $38(63.3 \%)$ & \\
\hline Mais de $1666 € /$ mês & $17(27.9 \%)$ & $17(28.3 \%)$ & \\
\hline \multicolumn{4}{|l|}{ Características clínicas } \\
\hline Tempo de diagnóstico, $n(\%)$ & & & $\chi_{(1)}^{2}=4.17^{*}$ \\
\hline Menos de 2 anos & $16(26.2 \%)$ & $7(11.7 \%)$ & \\
\hline Mais de 2 anos & 45 (73.8\%) & $53(88.3 \%)$ & \\
\hline Tipo de tratamentos & & & $\chi^{2}{ }_{(1)}=1.15$ \\
\hline Medicamentosos & $54(88.5 \%)$ & $50(83.3 \%)$ & \\
\hline Outros & $6(9.8 \%)$ & $10(16.7 \%)$ & \\
\hline Independência na administração dos tratamentos & & & $\chi^{2}(3)=0.68$ \\
\hline Sim & $54(88.5 \%)$ & $50(83.3 \%)$ & \\
\hline Não & 7 (11.5\%) & $10(16.7 \%)$ & \\
\hline
\end{tabular}

${ }^{*} p<.05 ;{ }^{* *} p<.01$, two-tailed.

Nos adultos emergentes, as doenças do aparelho respiratório (e.g., asma, rinite alérgica) e as doenças endócrinas, nutricionais e metabólicas (e.g., diabetes, hipotiroidismo) foram as mais frequentes (39.3\% e 27.9\%, respetivamente), enquanto nos adultos as doenças endócrinas, nutricionais e metabólicas mostraram ser as mais prevalentes $(31.7 \%)$, seguindo-se pelas doenças do sistema osteomuscular e do tecido conjuntivo (e.g., artrite reumatóide, osteoporose; $20.0 \%$ ). A comparação entre os grupos etários revelou homogeneidade em relação às características sociodemográficas e clínicas, com exceção do tempo de diagnóstico, situação profissional e estado civil. No entanto, a maior percentagem de adultos emergentes estudantes e solteiros e a maior percentagem de adultos trabalhadores e casados ou a viver em união de facto eram expetáveis, visto que se referem a características relacionadas com as tarefas desenvolvimentais inerentes a cada um destes grupos etários e, portanto, não foram controladas nas análises posteriores. 


\section{Variáveis e Instrumentos}

\section{Locus de controlo}

O locus de controlo foi avaliado através da Forma A da Multidimensional Health Locus of Control Scale (MHLC; Wallston, 2005). Esta escala é composta por 18 itens, respondidos numa escala de Likert de 5 pontos ( 1 = Discordo fortemente a 5 = Concordo fortemente), que visam medir três dimensões, com 6 itens cada: locus de controlo interno, locus de controlo externo - acaso, e locus de controlo externo - outros poderosos. A dimensão de locus de controlo interno refere-se à crença de que a saúde é determinada pelos comportamentos do próprio indivíduo (e.g., "Se eu ficar doente, é o meu próprio comportamento que determina quão cedo eu voltarei a ficar bem."); a subescala de locus de controlo externo - acaso avalia a medida em que o indivíduo acredita que a sua saúde depende da sorte ou destino (e.g., "A maior parte das coisas que afetam a minha saúde acontecem-me por acaso."); e a subescala de locus de controlo externo - outros poderosos avalia a crença de que a saúde é determinada por outras pessoas como médicos, professores, ou familiares (e.g., "Ter contato frequente com o meu médico é a melhor forma de eu evitar ficar doente."; Almeida \& Pereira, 2006). Os resultados são obtidos através da média das pontuações dos 6 itens correspondentes a cada subescala, sendo que, quanto maior a pontuação, maior a crença de que a respetiva dimensão controla a sua saúde.

A MHLC tem demonstrado boa consistência interna, tanto no estudo original ( $\alpha$ de Cronbach entre .67 e .77; Wallston, Wallston, \& DeVellis, 1978), como em estudos posteriores com versões traduzidas (e.g., $\alpha$ de Cronbach entre .53 e .72 na tradução espanhola validada na Colômbia; Rodríguez-Rosero, Ferriani, \& Coleta, 2002). No presente estudo, a MHLC apresentou uma consistência interna aceitável, com um $\alpha$ de Cronbach, para o grupo dos adultos emergentes e dos adultos, respetivamente, de .86 e .73 para a dimensão de locus de controlo interno; $\alpha=.63$ e .65 para a dimensão de locus de controlo externo - acaso; e $\alpha=.66$ e .64, para a dimensão de locus de controlo externo - outros poderosos.

\section{Adesão terapêutica}

A Medida de Adesão aos Tratamentos (MAT; Delgado \& Lima, 2001) foi empregada para avaliar a adesão terapêutica. A MAT é um questionário de autorresposta unidimensional, composto por 7 itens (e.g., "Alguma vez se esqueceu de tomar os medicamentos para a sua doença?"), respondidos numa escala de tipo Likert entre 1 (Sempre) e 6 (Nunca). A pontuação total é calculada pela média dos 7 itens, sendo que uma pontuação mais elevada indica maiores níveis de adesão aos tratamentos. A escala apresentou boas qualidades psicométricas no estudo original, com um $\alpha$ de Cronbach de .74 e validade concorrente com o nível de adesão aos tratamentos calculado a partir do rácio entre o número de medicamentos efetivamente tomados e o número de medicamentos prescritos (Delgado \& Lima, 2001). Na nossa amostra, a MAT apresentou um $\alpha$ de Cronbach de .84 para o grupo dos adultos emergentes e .78 para o grupo dos adultos, confirmando a fiabilidade do instrumento.

\section{Variáveis clínicas e sociodemográficas}

Os participantes preencheram ainda um questionário de dados sociodemográficos (e.g., idade, sexo, estado civil, escolaridade, profissão) e clínicos (e.g., estado de saúde, regime terapêutico, acompanhamento psicológico/psiquiátrico), desenvolvido especificamente para este projeto.

\section{Análises Estatísticas}

As análises de dados foram efetuadas através do programa de tratamento estatístico Statistical Package for the Social Sciences, versão 22.0 (IBM Corp., Armonk, NY). À exceção dos dados clínicos e sociodemográficos, os missing-values, que eram aleatórios e constituíam menos de $20 \%$ dos valores, foram tratados por substituição pela pontuação média individual para cada uma das variáveis. As análises estatísticas foram efetuadas a um intervalo de confiança de 95\%, exceto para o nível de significância das análises de regressão, onde foi considerado um nível de significância de $p<.10$, de forma a compensar a perda de poder estatístico inerente às interações introduzidas nas análises de regressão múltipla (Aguinis, 1995).

Para efeitos de caracterização da amostra, foram calculadas estatísticas descritivas para as variáveis clínicas e sociodemográficas e a homogeneidade das características da amostra entre os dois grupos etários foi analisada através de testes $t$ de Student para amostras independentes (variáveis contínuas) e testes de Qui-quadrado (variáveis categoriais). As diferenças nos valores médios das dimensões do locus de controlo e da adesão terapêutica entre os grupos etários foram analisadas, respetivamente, através de análises multi- e univariadas da covariância [(M)ANCOVA], controlando o tempo de diagnóstico. 
Previamente à realização das análises de regressão, foram calculados os coeficientes de correlação de Pearson entre as variáveis em estudo para avaliar as associações entre elas. As variáveis clínicas e/ou sociodemográficas que estavam correlacionadas com a variável dependente (i.e., adesão terapêutica) foram incluídas como covariáveis no modelo de regressão (MacKinnon \& Luecken, 2008). Para reduzir a multicolinearidade com os termos de interação e para tornar os coeficientes de regressão mais significativos e interpretáveis, as variáveis independentes (locus de controlo interno, locus de controlo externo - acaso e locus de controlo externo - outros poderosos) foram centradas antes de serem calculados os termos de interação (Aiken \& West, 1991). O papel moderador do grupo etário na relação entre as dimensões do locus de controlo e a adesão terapêutica foi examinado através de uma regressão linear hierárquica, incluindo no primeiro bloco as covariáveis, no segundo bloco as dimensões do locus de controlo centradas (variáveis independentes), no terceiro bloco o grupo etário (variável moderadora) e no quarto bloco os termos de interação entre as variáveis independentes e a variável moderadora. Usando a ferramenta computacional Modgraph (Jose, 2013), os efeitos de interação significativos foram representados graficamente em relação às estimativas da variável dependente nos diferentes valores do moderador e a força e significância estatística de cada linha de regressão foram analisadas através de testes post-hoc de análise do declive das retas de regressão.

\section{RESULTADOS}

\section{Análises Comparativas do Locus de Controlo e Adesão Terapêutica entre Grupos Etários}

A Tabela 2 apresenta as estatísticas descritivas para as dimensões do locus de controlo e adesão terapêutica e os resultados das análises univariadas. A MANCOVA para as dimensões do locus de controlo mostrou que não existem diferenças significativas entre os grupos etários, Wilks' Lambda $=.95 ; F_{(3,116)}=$ 2.17, $p=.10 ; \eta_{\mathrm{p}}{ }^{2}=.05$. Também não foram encontradas diferenças estatisticamente significativas entre adultos emergentes e adultos em relação à adesão terapêutica, $F_{(1,118)}=0.17, p=.68 ; \mathrm{y}_{\mathrm{p}}{ }^{2}<.01$.

Tabela 2. Análises comparativas do locus de controlo e adesão terapêutica entre grupos etários.

\begin{tabular}{|c|c|c|c|}
\hline & $\begin{array}{l}\text { Adultos emergentes } \\
\qquad(n=61)\end{array}$ & $\begin{array}{l}\text { Adultos } \\
(n=60)\end{array}$ & $\begin{array}{c}\text { Diferenças entre } \\
\text { grupos }\end{array}$ \\
\hline & $M(D P)$ & $M(D P)$ & $F_{(1,118)}$ \\
\hline LC interno & $3.75(1.08)$ & $3.97(0.81)$ & 2.15 \\
\hline LC externo - acaso & $2.35(0.69)$ & $2.60(0.83)$ & 3.37 \\
\hline LC externo - outros poderosos & $3.33(0.90)$ & $3.63(0.83)$ & 3.87 \\
\hline Adesão terapêutica & $4.99(0.76)$ & $4.99(0.67)$ & 0.17 \\
\hline
\end{tabular}

$p<.05{ }^{* *} p<.01$, two-tailed.

Efeitos Principais e de Interação do Locus de Controlo e do Grupo Etário na Adesão Terapêutica Conforme apresentado na Tabela 3, as análises de correlação preliminares indicaram uma associação negativa entre o locus de controlo externo - acaso e a adesão terapêutica.

Tabela 3. Correlações entre o locus de controlo, a adesão terapêutica e as variáveis clínicas e sociodemográficas.

\begin{tabular}{|c|c|c|c|c|}
\hline & LC interno & $\begin{array}{l}\text { LC externo - } \\
\text { acaso }\end{array}$ & $\begin{array}{c}\text { LC externo -outros } \\
\text { poderosos }\end{array}$ & $\begin{array}{c}\text { Adesão } \\
\text { terapêutica }\end{array}$ \\
\hline LC interno & - & & & \\
\hline LC externo - acaso & .09 & - & & \\
\hline LC externo - outros poderosos & $.30^{* *}$ & $.30^{* *}$ & - & \\
\hline Adesão terapêutica & -.12 & $-.23^{* *}$ & .06 & - \\
\hline Grupo etário a $^{a}$ & .12 & .17 & .17 & $<-.01$ \\
\hline Sexo $^{b}$ & $<.01$ & -.13 & -.04 & .03 \\
\hline Escolaridade em anos & -.01 & .06 & -.12 & .04 \\
\hline 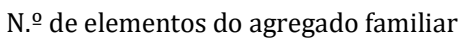 & $-.33^{* *}$ & -.02 & -.14 & -.16 \\
\hline Rendimento do agregado familiar ${ }^{c}$ & -.13 & -.09 & -.03 & .04 \\
\hline Tempo de diagnóstico ${ }^{\mathrm{d}}$ & -.09 & .02 & -.02 & $-.21^{*}$ \\
\hline Tipo de tratamentos e & .11 & -.09 & .11 & .08 \\
\hline $\begin{array}{l}\text { Independência na administração dos } \\
\text { tratamentos }\end{array}$ & -.03 & -.11 & -.11 & .02 \\
\hline
\end{tabular}

${ }^{a}$ Grupo de referência: 0 = Adultos emergentes; ${ }^{\mathrm{b}}$ Grupo de referência: 0 = Masculino. ${ }^{\mathrm{c}}$ Grupo de referência: $0=$ Rendimento ilíquido

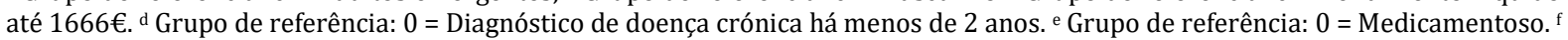
Grupo de referência: $0=$ Sim. ${ }^{*} p<.05 ;{ }^{* *} p<.01$, two-tailed. 
A dimensão de locus de controlo externo - outros poderosos associou-se, positiva e significativamente, com o locus de controlo interno e com o locus de controlo externo - acaso. Em relação às associações com as variáveis sociodemográficas e clínicas, foram encontradas correlações significativas entre um menor número de elementos do agregado familiar e maior tendência para um locus de controlo interno da saúde, e entre maior tempo de diagnóstico e menor adesão terapêutica.

Os resultados da análise de regressão hierárquica para testar os efeitos principais e de interação das dimensões do locus de controlo e do grupo etário na adesão terapêutica, controlando o efeito do tempo de diagnóstico (por estar significativamente associado à variável dependente, i.e., à adesão terapêutica) são apresentados na Tabela 4.

Tabela 4. Efeitos principais e de interação do locus de controlo e do grupo etário na adesão terapêutica.

\begin{tabular}{|c|c|c|c|c|c|c|c|c|}
\hline \multirow{3}{*}{ Variáveis } & \multirow{2}{*}{\multicolumn{2}{|c|}{$\begin{array}{c}\text { Bloco 1: Covariáveis } \\
\qquad \begin{array}{c}\Delta R^{2}=.04 ; \\
\Delta \boldsymbol{F}_{(\mathbf{1}, \mathbf{1 1 9})}=\mathbf{5 . 2 2 ^ { * * }}\end{array}\end{array}$}} & \multirow{2}{*}{\multicolumn{2}{|c|}{$\begin{array}{c}\text { Bloco 2: Efeitos } \\
\text { principais do locus de } \\
\text { controlo } \\
\Delta R^{2}=.09 ; \\
\Delta \boldsymbol{F}_{(\mathbf{3}, \mathbf{1 1 6})}=\mathbf{4 . 1 9 ^ { * * * }}\end{array}$}} & \multirow{2}{*}{\multicolumn{2}{|c|}{$\begin{array}{c}\text { Bloco 3: Efeito } \\
\text { principal do grupo } \\
\text { etário } \\
\Delta R^{2}=.01 ; \\
\Delta \boldsymbol{F}_{(\mathbf{1}, \mathbf{1 1 5})}=\mathbf{0 . 7 3}\end{array}$}} & \multirow{2}{*}{\multicolumn{2}{|c|}{$\begin{array}{c}\text { Bloco 4: Efeitos de } \\
\text { interação entre o LC e } \\
\text { o grupo etário } \\
\Delta R^{2}=.05 ; \\
\Delta \boldsymbol{F}_{(\mathbf{3 , 1 1 2})}=\mathbf{2 . 3 0 ^ { * }}\end{array}$}} \\
\hline & & & & & & & & \\
\hline & $b(S E)$ & $t$ & $b(S E)$ & $t$ & $b(S E)$ & $t$ & $b(S E)$ & $t$ \\
\hline Tempo de diagnóstico $^{\mathrm{a}}$ & $-.37(.16)$ & $-2.28^{* *}$ & $-.39(.16)$ & $-2.46^{* *}$ & $-.41(.16)$ & $-2.57^{* *}$ & $-.41(.16)$ & $-2.62^{*}$ \\
\hline LC interno & & & $-.13(.07)$ & $-1.90^{*}$ & $-.13(.07)$ & $-1.97^{* *}$ & $-.28(.09)$ & $-3.09^{* * *}$ \\
\hline LC externo - acaso & & & $-.25(.08)$ & $-2.94^{* * *}$ & $-.25(.08)$ & $-3.01^{* * *}$ & $-.15(.14)$ & -1.08 \\
\hline $\begin{array}{l}\text { LC externo - outros } \\
\text { poderosos }\end{array}$ & & & $.15(.08)$ & $1.93^{*}$ & $.14(.08)$ & $1.82^{*}$ & $.28(.11)$ & $2.69^{* * *}$ \\
\hline Grupo etário b & & & & & $.11(.13)$ & 0.85 & $.09(.13)$ & 0.71 \\
\hline LC interno* Grupo etário & & & & & & & $.31(.15)$ & $2.12^{* *}$ \\
\hline $\begin{array}{l}\text { LC externo - acaso * Grupo } \\
\text { etário }\end{array}$ & & & & & & & $-.08(.18)$ & -0.44 \\
\hline $\begin{array}{l}\text { LC externo - outros } \\
\text { poderosos * Grupo etário }\end{array}$ & & & & & & & $-.28(.15)$ & $-1.83^{*}$ \\
\hline Resumo do modelo & $R^{2}=.04 ; F$ & $=5.22^{* *}$ & $\begin{array}{r}R^{2}=.14 \\
4\end{array}$ & $4,116)=$ & $\begin{array}{r}R^{2}=.14 \\
3 .\end{array}$ & $5,115)=$ & $R^{2}=.19$ & $8,112)=$ \\
\hline
\end{tabular}

${ }^{\mathrm{a}}$ Grupo de referência: 0 = Diagnóstico de doença crónica há menos de 2 anos; ${ }^{\mathrm{b}}$ Grupo de referência: 0 = Adultos emergentes.

${ }^{*} p<.10 ;{ }^{* *} p<.05 ;{ }^{* * *} p<.01$, two-tailed.

Controlando o tempo de diagnóstico, foram encontrados efeitos principais significativos das várias dimensões do locus de controlo, que explicaram $9 \%$ da variância da adesão ao tratamento. Especificamente, uma maior tendência para apresentar um locus de controlo interno ou externo fundamentado na crença de que a saúde depende do acaso, associou-se a menor adesão terapêutica, enquanto uma maior tendência para uma orientação de locus de controlo externo fundamentado na crença de que a saúde depende da ação de outros poderosos, associou-se a maior adesão terapêutica. 0 grupo etário explicou uma proporção inferior a $1 \%$ da variância dos níveis de adesão terapêutica. No entanto, os termos de interação "locus de controlo interno * grupo etário" e "locus de controlo externo outros poderosos * grupo etário" explicaram uma variância adicional significativa de 5\% na adesão terapêutica.

Os testes post-hoc de análise do declive das retas de regressão (Figuras 1 e 2) revelaram que ambas as associações entre maior locus de controlo interno e menor adesão terapêutica e entre maior locus de controlo externo - outros poderosos e maior adesão terapêutica foram significativas apenas para o grupo dos adultos emergentes $(b=-.28$, SE $=.09 ; t=-3.15, p<.01$ e $b=.28$, SE $=.10 ; t=2.69, p<.01$, respetivamente), mas não significativas para o grupo dos adultos $(b=.03$, SE $=.11 ; t=0.24, p=.81$ e $b<$ $.01, \mathrm{SE}=.11 ; t=0.03, p=.98$, respetivamente). 
Reis, Pires \& Silva

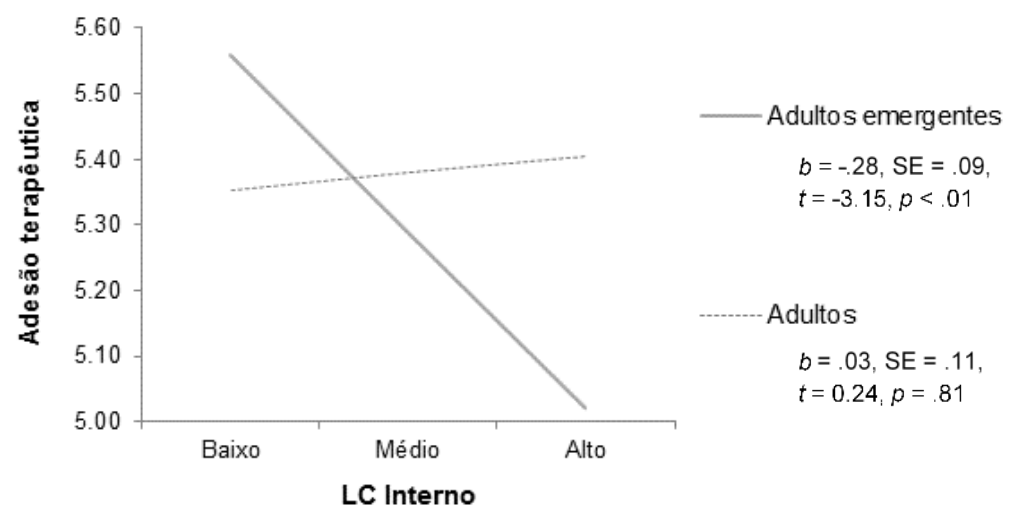

Figura 1. Efeito moderador do grupo etário na relação entre locus de controlo interno e adesão terapêutica

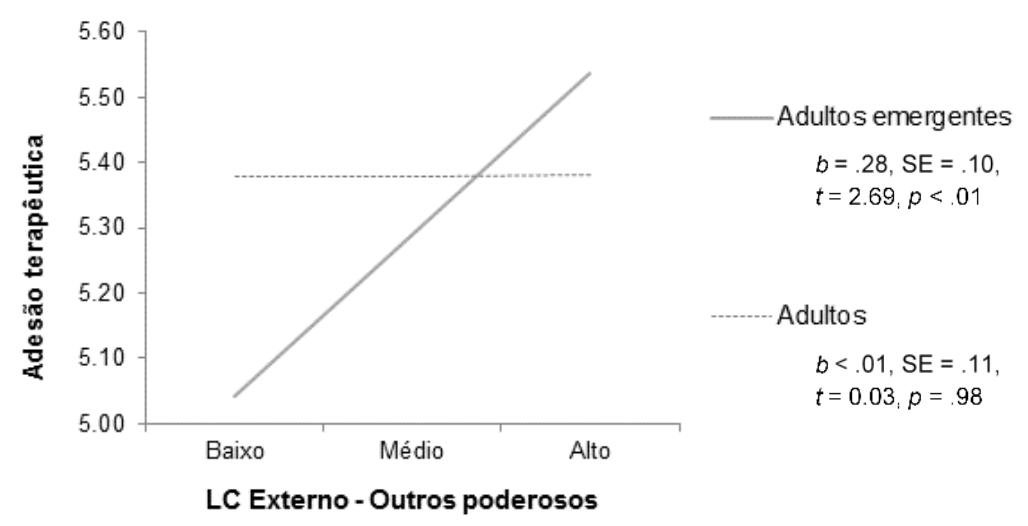

Figura 2. Efeito moderador do grupo etário na relação entre locus de controlo externo - outros poderosos e adesão terapêutica

\section{DISCUSSÃO}

Este trabalho investigou o locus de controlo da saúde e a adesão terapêutica em diferentes grupos etários (i.e., adultos emergentes e adultos) com condições crónicas de saúde e o papel moderador do grupo etário nas associações entre o locus de controlo e a adesão aos tratamentos. Os resultados mostraram que não existem diferenças significativas na orientação do locus de controlo nem nos níveis de adesão aos tratamentos entre os dois grupos etários estudados. No entanto, observaram-se diferenças na força das associações, negativa e positiva, respetivamente, entre o locus de controlo interno e externo - outros poderosos e a adesão terapêutica, sendo estas associações significativas apenas para o grupo dos adultos emergentes, enquanto o locus de controlo externo - acaso associou-se negativamente com a adesão terapêutica independentemente da idade dos participantes.

Embora fosse esperada uma maior adesão aos tratamentos no grupo dos adultos (H1), em comparação com os mais jovens, esta hipótese não foi confirmada pelos resultados do estudo. Ao falar da adesão terapêutica em condições crónicas de saúde, a literatura menciona que existem vários fatores que a podem influenciar, para além do grupo etário. As características associadas ao tratamento (Williams, Manias, \& Walker, 2008), nomeadamente a sua complexidade, constitui um dos principais fatores que podem contribuir para a não adesão (Hansen, Seifeldin, \& Noe, 2007; Haynes, McDonald, \& Garg, 2002; Yeager et al., 2005). Os estudos que analisam o impacto dos diferentes regimes de tratamento na adesão terapêutica concluíram que as percentagens de adesão decrescem quando os regimes são mais complexos e requerem alterações no estilo de vida (Martin, Williams, Haskard, \& DiMatteo, 2005), em comparação com os tratamentos apenas medicamentosos (com taxas de adesão de $33 \%$ e 50\%, respetivamente). Tratamentos que impliquem mudanças no estilo de vida são mais complexos e requerem a sensibilização, educação e apoio por parte dos profissionais de saúde, pois estes são difíceis de implementar e manter a longo prazo (Pollack, Chastek, Williams, \& Moran, 2010). Sawicki e Tiddens (2012) referem que a complexidade dos tratamentos influencia negativamente a adesão tanto nos adolescentes, pois implica que eles tenham de assumir a responsabilidade pelos seus próprios cuidados, como nos adultos, porque estes têm de equilibrar o tratamento e a gestão da sua doença com as obrigações familiares, educacionais e laborais. A inexistência de diferenças nos níveis de adesão entre os grupos etários observados no 
presente estudo pode assim ser devida ao facto de a maioria dos participantes, em ambos os grupos etários, estarem apenas a realizar tratamentos medicamentosos para a sua condição de saúde.

Em relação às crenças de controlo da saúde, não foram encontradas diferenças entre os adultos emergentes e os adultos. Berglund e colaboradores (2014) referem que o locus de controlo tem tendência para estabilizar ao longo do tempo, não sendo fácil de mudar. As perceções de controlo tendem a estabilizar durante a idade adulta, só havendo uma diminuição das mesmas na transição para a terceira idade, estando estas associadas ao aumento da conscientização das limitações pessoais para lidar com situações percebidas como menos controláveis e menos contingentes à ação do próprio indivíduo (Lachman et al., 2011). Dadas as características clínicas da população estudada nesta investigação, i.e., o facto dos participantes de ambos os grupos etários estarem a lidar com uma doença crónica enquanto acontecimento indutor de stresse, pode explicar esta ausência de diferenças na orientação predominante do locus de controlo, uma vez que o locus de controlo pode ser influenciado tanto pelas características particulares dos próprios indivíduos, como pela natureza da situação vivenciada (Rotter, 1975).

A única dimensão do locus de controlo que se associou significativa e negativamente à adesão terapêutica em ambos os grupos etários foi o locus de controlo externo - acaso, confirmando a terceira hipótese do estudo. Outros autores também têm encontrado resultados semelhantes, referindo que este tipo de orientação de controlo está associado a resultados de saúde mais pobres, particularmente em situações de doença crónica, uma vez que está relacionado com falsas esperanças (Aflakseir \& ZarrinPour, 2013). O locus de controlo externo - acaso aumenta o risco de resultados de saúde negativos, uma vez que os indivíduos tendem a acreditar que o acaso controla a progressão da sua doença, prejudicando assim a aprendizagem de habilidades de autogestão de saúde (Ferris \& Mahan, 2009). Assim, os indivíduos tendem a desvalorizar a importância que o seu próprio comportamento e que as ações dos outros (familiares, profissionais de saúde, etc.) podem ter sobre a sua saúde. Esta crença de controlo pode também prejudicar o desenvolvimento da confiança que é depositada nos prestadores de cuidados de saúde (Brinks, Feaster, Burns, \& Mitrani, 2010). A invariância desta associação entre os grupos estudados (adultos emergentes vs. adultos) pode ser entendida pela equipolência das situações de saúde vivenciadas. De acordo com Aflakseir e ZarrinPour (2013), uma orientação de locus de controlo externo acaso só poderá ser adaptativa perante uma doença considerada terminal, pois esta condição encontra-se completamente fora do controlo de qualquer ação pessoal ou médica, que não é o caso da situação clínica dos participantes do presente estudo.

Apesar de não terem sido encontradas diferenças nas tendências de locus de controlo da saúde entre grupos etários, a importância atribuída ao locus de controlo interno e externo - outros poderosos, enquanto fatores explicativos da variância na adesão terapêutica diferiu consoante o grupo etário, ganhando mais relevância no grupo dos jovens adultos. Estes resultados refutam a segunda hipótese do estudo, que previa associações positivas entre uma maior tendência para um locus de controlo interno ou externo - outros poderosos, estivesse associada a maior adesão terapêutica. Nos adultos, os níveis de adesão terapêutica não apresentaram grandes variações em função da orientação de locus de controlo. São escassos os estudos que reportam ausência de influência do locus de controlo na adesão aos regimes terapêuticos. Wall (1992), ao chegar a estes resultados, justificou-os com base na multiplicidade de fatores que poderão interferir na relação entre estas duas variáveis e que não foram contempladas e controladas neste estudo, como é o caso da complexidade do tratamento e gravidade da doença.

No grupo de adultos emergentes, verificou-se uma associação entre maior locus de controlo externo - outros poderosos e maior adesão terapêutica. Estudos prévios têm mostrado igualmente que uma orientação mais externa de controlo pode ter um efeito positivo na adesão (Almeida \& Pereira, 2006; Burish, Carey, Wallston, Stein, Jamison, \& Lyles, 1984). Se, por um lado, a adolescência é caracterizada pela procura ativa de independência em relação aos pais e aquisição da perceção de controlo sobre as suas vidas, a vivência de stressores adicionais decorrentes da doença crónica pode originar a perceção de falta de controlo sobre os acontecimentos e ações pessoais (Almeida \& Pereira, 2006; Taddeo et al., 2008). Assim, poderá ser importante fomentar a confiança nos pais ou outros cuidadores familiares ou nos profissionais de saúde enquanto agentes externos que podem ajudar na gestão da saúde dos jovens.

Embora alguma literatura reforce a importância de adquirir um locus de controlo orientado para a internalidade na fase de transição para a idade adulta, particularmente, perante uma condição crónica de saúde, os resultados deste estudo mostraram uma associação negativa entre o locus de controlo interno e a adesão terapêutica, apenas no grupo dos adultos emergentes. Wortman e Dunkel-Schetter (1979) referem que os doentes crónicos com um locus de controlo interno podem vir a desenvolver sentimentos de frustração e impotência devido à sensação de incapacidade perante a condição de saúde que vivenciam, resultando numa menor realização de comportamentos de adesão (Strickland, 1989). Por outro lado, os indivíduos como uma orientação mais externa poderão sentir-se mais vulneráveis, acabando por serem mais recetivos ao aconselhamento de profissionais de saúde e estando mais 
propensos a desempenhar um papel ativo na promoção da sua saúde (Burish et al., 1984), aderindo assim a mais comportamentos de controlo sobre a sua doença e seguindo mais estritamente os tratamentos (Almeida \& Pereira, 2006). Esta ideia é reforçada por Jamison e colaboradores (1986), que argumentam que a adoção de uma orientação de controlo externo, fundamentada na confiança nos profissionais de saúde, pode ser adaptativa para os jovens que vivenciam uma situação de doença crónica uma vez que estes são confrontados com uma situação com pouca possibilidade de controlo, prejudicando assim o desenvolvimento e/ou manutenção de crenças de controlo pessoal (Almeida \& Pereira, 2006). Neste contexto, esta externalidade poderá ajudar a reduzir o sofrimento relacionado com a perda de controlo quando os adultos emergentes se tornam conscientes do aumento da sua dependência em relação a estes profissionais ou outras pessoas para as decisões relacionadas com a sua saúde (Jamison et al., 1986).

\section{Limitações do Estudo}

Os resultados deste estudo devem ser interpretados tendo em conta algumas limitações metodológicas. Em primeiro lugar, o seu desenho transversal impede o estabelecimento de relações de causalidade entre as variáveis, nomeadamente entre as dimensões do locus de controlo e a adesão terapêutica. Em segundo lugar, a dimensão reduzida e a seleção não probabilística por conveniência da amostra dificultam a generalização dos resultados para a população de doentes crónicos. Alguns autores referem que o locus de controlo pode ser influenciado pelas características da situação (e.g., tipo de doença; Rotter, 1975) e que os níveis de adesão terapêutica estão associados às características do tratamento (e.g., complexidade dos tratamentos; Sawicki \& Tiddens, 2012; Williams et al., 2008). No entanto, o método de recolha da amostra utilizado nesta investigação deu origem a uma grande diversidade de diagnósticos e de regimes terapêuticos, impedindo a análise dos efeitos específicos destas variáveis no locus de controlo e na adesão terapêutica. Desta forma, o presente estudo adotou uma perspetiva não categorial, com base no argumento de que as condições crónicas de saúde, na sua generalidade, podem originar limitações funcionais, dependência de mecanismos compensatórios para minimizar essas limitações e recurso ou necessidade acrescida de cuidados, médicos ou outros (Silver, Westbrook, \& Stein, 1998), conduzindo assim a mais similitudes do que disparidades nos resultados de adaptação. Uma outra limitação diz respeito à heterogeneidade demográfica, identitária e subjetiva que caracteriza o grupo dos adultos emergentes (Arnett, 2000) e à variabilidade de idades que marcam o final desta etapa desenvolvimental. No presente estudo, a média de idades do grupo dos adultos foi relativamente baixa $(M=37.93 ; D P=$ 8.75), podendo justificar a ausência de diferenças no locus de controlo e nos níveis de adesão em relação aos adultos emergentes. Adicionalmente, e apesar do reconhecimento de que o grupo dos adultos incluiu uma grande variabilidade etária, a dimensão reduzida da amostra $(n=60)$ impossibilitou a análise das especificidades das diferentes fases do ciclo de vida (e.g., adultos jovens, adultos de meia-idade). Estudos futuros deverão replicar estes resultados utilizando outros grupos etários como controlos, nomeadamente adultos de meia-idade e idosos. Por fim, deve ser referida a inexistência de estudos psicométricos da forma A do instrumento de avaliação do locus de controlo (Multidimensional Health Locus of Control Scale) para a população portuguesa, existindo apenas um estudo que utiliza o instrumento (forma C) numa população de adultos portugueses com idades iguais ou superiores a 50 anos (Nunes \& Tapadinhas, 2014).

\section{Implicações para a Prática Clínica e Investigação}

Os resultados deste estudo sugerem alguma adequação para retratar mecanismos comuns de adaptação e formas de lidar com o stresse e podem ser úteis para orientar e facilitar rotinas de avaliação e intervenção psicossocial nos contextos de saúde. Existe uma grande discrepância em relação à investigação e à prática clínica nesta área. Assim, é importante implementar intervenções que tenham em atenção as avaliações que os indivíduos fazem das situações (Ridder \& Schreurs, 2001). O locus de controlo poderá estar associado a resultados de adaptação mais ou menos positivos. Se, por um lado, modificar crenças de controlo pode ser mais difícil do que alterar outros fatores que concorrem para a não adesão, por outro lado, devem ser dadas informações aos pacientes acerca da diversidade e benefícios de cada intervenção disponível e ajudá-los a selecionar as que têm uma maior probabilidade de sucesso (Almeida \& Pereira, 2006; Cameron, 1996). Estas medidas reforçariam a perceção realista acerca do tratamento (Cameron, 1996) e dos seus benefícios a longo prazo (Sherbourne, Hays, Ordway, DiMatteo, \& Kravitz, 1992), bem como a diminuição da crença de que a sua saúde depende do acaso, da sorte ou do destino, aumentando assim a probabilidade do cumprimento das prescrições terapêuticas. Ao disponibilizar informações claras sobre os diversos tratamentos, formas de o seguir e os recursos disponíveis, a perceção da ameaça será reduzida e a motivação para fazer algo será maior (Cameron, 1996). Os resultados deste estudo sugerem que estas intervenções psicoeducativas com vista à redução das atribuições de controlo ao acaso poderão ser eficazes em ambos os grupos etários. 
A principal implicação dos resultados deste estudo prende-se com a importância da adequação das intervenções sobre o locus de controlo interno e externo - outros poderosos, por parte de equipas de saúde multidisciplinares (nas quais deverão estar inseridos psicólogos), em conformidade com as especificidades da faixa etária (Ridder \& Schreurs, 2001), a fim de promover, efetivamente, resultados de adaptação mais positivos, como é o caso da adesão terapêutica. Assim, a etapa desenvolvimental de transição para a idade adulta poderá beneficiar de intervenções priorizadas que visem reduzir o impacto e os encargos adicionais que a gestão de uma condição crónica de saúde e dos seus tratamentos detêm sobre os aspetos desenvolvimentais e processos de formação da identidade característicos dos adultos emergentes (Evan, Kaufman, Cool, \& Zelter, 2006).

Os resultados deste estudo mostram que os adultos emergentes, embora não apresentem diferenças nos níveis de adesão terapêutica em comparação com os adultos mais velhos, são um grupo em que uma orientação de locus de controlo externo, fundamentado na confiança nos profissionais de saúde, pode ter mais influência nos seus padrões de adesão. Portanto, aquando de intervenções em que o alvo terapêutico seja o locus de controlo, os adultos emergentes devem ser priorizados, uma vez que a relação entre o locus de controlo e adesão terapêutica é mais forte nesta faixa etária. Para promover a adesão nestes indivíduos, é importante sensibilizar os profissionais de saúde para as capacidades de comunicação, de forma a estarem atentos às necessidades específicas dos seus pacientes e personalizarem os seus tratamentos, uma vez que, para os adultos emergentes, a sua doença constitui apenas um aspeto das suas vidas, sendo que estes também apresentam outras necessidades, prioridades e papéis sociais (Hansen et al., 2007; Kyngäs, 2000). As intervenções que têm por base a negociação são importantes com esta população porque, para além de dotarem os indivíduos de algum domínio, diminuem a sensação de que a sua doença e tratamentos os vão impedir de desempenhar as tarefas desenvolvimentais de transição para a idade adulta (Kyngäs, 2000). Desta forma, os profissionais de saúde que lidam com estes pacientes devem ser sensíveis à importância do trabalho de competências que incrementem a participação dos mesmos nos tratamentos prescritos (Cherepakho, 2006) e devem potenciar a modificação de crenças de controlo de forma a permitir a aquisição de novas ferramentas e comportamentos adequados e saudáveis para que os adultos emergentes possam lidar com a sua condição de saúde (Lachman et al., 2011). Esta modificação de crenças de controlo da saúde poderá resultar em melhores resultados de saúde, como é o caso da adesão às prescrições terapêuticas (Cherepakho, 2006).

Investigações futuras deverão examinar as associações propostas pelo modelo de Stresse e Coping de forma longitudinal e contemplar uma amostra mais representativa da população, assim como outras variáveis que poderão estar associadas à adesão terapêutica, nomeadamente, a gravidade da doença crónica e o tipo e complexidade dos tratamentos. Será também importante identificar as estratégias de coping que concorrem para uma maior adesão terapêutica e que medeiam a relação entre diferentes orientações de controlo e os resultados de saúde, de forma a melhor compreender a vivência e a adaptação a uma doença crónica e, posteriormente, permitir o desenvolvimento e validação empírica de estratégias de intervenção psicossociais mais eficazes e promotoras de resultados de saúde mais positivos.

\section{REFERÊNCIAS}

Aflakseir, A., \& ZarrinPour, R. (2013). Predicting adherence to diet regimen based on health locus of control: A cross sectional study. Irian Journal of Diabetes and Obesity, 5(2), 71-76.

Aguinis, H. (1995). Statistical power with moderated multiple regression in management research. Journal of Management, 21, 1141-1158. https://doi.org/10.1177/014920639502100607

Aiken, L., \& West, S. (1991). Multiple regression: Testing and interpreting interactions. Thousand Oaks, CA: Sage.

Aldwin, C. (1991). Does age affect the stress and coping process? Implications of age differences in perceived control. Journal of gerontology, 46, 174-180. https://doi.org/10.1093/geronj/46.4.P174

Almeida, J., \& Pereira, M. (2006). Locus de controlo na saúde: Conceito e validação duma escala em adolescentes com diabetes tipo I. Psicologia, Saúde \& Doenças, 7(2), 221-238.

American Psychological Association. (2010). Ethical principles of psychologists and code of conduct. Retirado de http://www.apa.org/ethics/code/principles.pdf

Anderson, B. J., \& Wolpert, H. A. (2004). A developmental perspective on the challenges of diabetes education and care during the young adult period. Patient Education and Counseling, 53, 347-352. https://doi.org/10.1016/j.pec.2003.03.001

Andrade, C. (2010). Transição para a idade adulta: Das condições sociais às implicações psicológicas. Análise Psicológica, 2, 255-267. https://doi.org/10.14417/ap.279

Arnett, J. J. (2000). Emerging adulthood: A theory of development from the late teens through the 
twenties. American Psychologist, 55, 469-480. https://doi.org/10.1037/0003-066X.55.5.469

Arnett, J. J. (2006). Emerging adulthood: Understanding the new way of coming of age. In J. Arnett \& L. Tanner (Eds.). Emerging adulthood in America: Coming of age in the 21 ${ }^{\text {st }}$ Century (pp. 3-19). Washington, DC: American Psychological Association.

Barletta, J. (2010). Comportamentos e crenças em saúde: Contribuições da psicologia para a medicina comportamental. Revista de Psicologia da IMED, 2, 307-317. https://doi.org/10.18256/21755027/psico-imed.v2n1p307-318

Berglund, E., Lytsy, P., \& Westerling, R. (2014). The influence of locus of control on self-rated health in context of chronic disease: A structural equation modeling approach in a cross sectional study. BMC Public Health, 14, 492. https://doi.org/10.1186/1471-2458-14-492

Blanchard-Fields, F., \& Robinson, S. (1987). Age differences in the relation between controllability and coping. Journal of Gerontology, 42, 497-501. https://doi.org/10.1093/geronj/42.5.497

Bleyer, W. (2002). Cancer in older adolescents and young adults: epidemiology, diagnosis, treatment, survival, and importance of clinical trials. Medical and Pediatric Oncology, 38, 1-10. https://doi.org/10.1002/mpo.1257

Blount, R., Simons, L., Devine, K., Jaaniste, T., Cohen, L., Chambers, C., \& Hayutin, L. (2007). Evidence-based assessment of coping and stress in pediatric psychology. Journal of Pediatric Psychology, 33, 10211045. https://doi.org/10.1093/jpepsy/jsm071

Blum, R., Garrel, D., Hodgman, C., Jorissen, T., Okinow, N., Orr, D., \& Slap, G. (1993). Transition from childcentered to adult health-care systems for adolescents with chronic conditions. A position paper of the Society for Adolescent Medicine. Journal of Adolescent Health, 14, 570-576. https://doi.org/10.1016/1054-139X(93)90143-D

Brincks, A. M., Feaster, D. J., Burns, M. J., \& Mitrani, U. B. (2010). The influence of health locus of control on the patient-provider relationship. Psychology, Health \& Medicine, 15, 720-728. https://doi.org/10.1080/13548506.2010.498921

Burish, T.., Carey, M.., Wallston, K.., Stein, M.., Jamison, R.., \& Lyles, J. (1984). Health locus of control and chronic disease: An external orientation may be advantageous. Journal of Social and Clinical Psychology, 2, 326-332. https://doi.org/10.1521/jscp.1984.2.4.326

Cameron, C. (1996). Patient compliance: Recognition of factors involved and suggestions for promoting compliance with therapeutic regimens. Journal of Advanced Nursing, 24, 244-250. https://doi.org/10.1046/j.1365-2648.1996.01993.x

Carrijo, R., \& Coleta, M. (2007). A influência de variáveis psicossociais na adesão ao tratamento de pacientes diabéticos. Horizonte Científico, 1(1), 1-25.

Cherepakho, V. (2006). Health locus of control, risk perception, and health behavior in African Americans. (Unpublished master's thesis). University of Pittsburgh, Pensilvânia, Estados Unidos. Retirado de http://d-scholarship.pitt.edu/6966/

Christensen, A., Wiebe, J., Benotsch, E., \& Lawton, W. (1996). Perceived health competence, health locus of control, and patient adherence in renal dialysis. Cognitive Therapy and Research, 20, 411-421. https://doi.org/10.1007/BF02228042

Delgado, A., \& Lima, M. L. (2001). Contributo para a validação concorrente de uma medida de adesão aos tratamentos. Psicologia, Saúde \& Doenças, 2(2), 81-100.

Dellmann-Jenkins, M., Blankemeyer, M., \& Pinkard, O. (2001). Incorporating the elder caregiving role into the developmental tasks of young adulthood. International Journal of Aging \& Human Development, 52,1-18. https://doi.org/10.2190/FGQA-65FU-JGNT-6C9J

Erickson, E. (1980). Identity and the life cycle. New York: Norton.

Evan, E., Kaufman, M., Cook, A., \& Zeltzer, L. (2006). Sexual health and self-esteem in adolescents and young adults with cancer. Cancer, 107, 1672-1679. https://doi.org/10.1002/cncr.22101

Ferris, M. E., Mahan, J. D. (2009). Pediatric chronic kidney disease and the process of health care $\begin{array}{llll}\text { transition. Seminars in Nephrology, } & \text { 29, } & \text { 435-444. }\end{array}$ https://doi.org/10.1016/j.semnephrol.2009.03.018

Fleming, J. S. (2008). Psychological perspectives on human developmental. Retirado de http://swppr.org/Textbook/Contents.html

Folkman, S. (1984). Personal control and stress and coping processes: A theoretical analysis. Journal of Personality and Social Psychology, 46, 839-852. https://doi.org/10.1037/0022-3514.46.4.839

Griva, K., Myers, L., \& Newman, S. (2000). Illness perceptions and self-efficacy beliefs in adolescents and young adults with insulin dependent diabetes mellitus. Psychology \& Health, 15, 733-750. https://doi.org/10.1080/08870440008405578

Hansen, R., Seifeldin, R., \& Noe, L. (2007). Medication adherence in chronic disease: Issues in posttransplant immunosuppression. Transplantation Proceeding, 39, 1287-1300. 
https://doi.org/10.1016/j.transproceed.2007.02.074

Haynes, R., McDonald, H., \& Garg, A. (2002). Helping patients follow prescribed treatment: Clinical applications. JAMA: Journal of the American Medical Association, 288, 2880-2883. https://doi.org/10.1001/jama.288.22.2880

Hesselink, A., Penninx, B., Schlösser, M., Wijnhoven, H., van der Windt, D., Kriegsman, D., \& van Eijk, J. (2004). The role of coping resources and coping style in quality of life of patients with asthma or COPD. Quality of Life Research, 13, 509-518. https://doi.org/10.1023/B:QURE.0000018474.14094.2f

Hinds, P., Quargnenti, A., Bush, A., Pratt, C., Fairclough, D., Rissmiller, G., ... Gilchrist, G. (2000). An evaluation of the impact of a self-care coping intervention on psychological and clinical outcomes in adolescents with newly diagnosed cancer. European Journal of Oncology Nursing, 4, 6-17. https://doi.org/10.1054/ejon.1999.0051

Jamison, R., Lewis, S., \& Burish, T. (1986). Psychological impact of cancer on adolescents: Self-image, locus of control, perception of illness and knowledge of cancer. Journal of Chronic Diseases, 39, 609-617. https://doi.org/10.1016/0021-9681(86)90186-4

Jose, P. (2013). ModGraph-I: A programme to compute cell means for the graphical display of moderational analyses: The internet version. New Zealand: Victoria University of Wellington. Retirado de https://psychology.victoria.ac.nz/modgraph/modgraph.php

Katz, P., Yelin, E., Eisner, M., \& Blanc, P. (2002). Perceived control of asthma and quality of life among adults with asthma. Annals of Allergy, Asthma, \& Immunology, 89, 251-258. https://doi.org/10.1016/S1081-1206(10)61951-5

Kellerman, J., Zeltzer, L., Ellenberg, L., Dash, J., \& Rigler, D. (1980). Psychological effects of illness in adolescence I: Anxiety, self-esteem, and perception of control. The Journal of Pediatrics, 97, 126131. https://doi.org/10.1016/S0022-3476(80)80152-1

Kelly, A. M., Kratz, B., Bielski, M., \& Rinehart, P. M. (2002). Implementing transitions for youth with complex chronic conditions using the medical home model. Pediatrics, 110(S3), 1322-1327.

Kondryn, H., Edmondson, C., Hill, J., \& Eden, T. (2011). Treatment non-adherence in teenage and young adult patients with cancer. The Lancet Oncology, 12, 100-108. https://doi.org/10.1016/S14702045(10)70069-3

Kurita, G., \& Pimenta, C. (2004). Adesão ao tratamento da dor crônica e o locus de controle da saúde. Revista da Escola de Enfermagem da USP, 38, 254-261. https://doi.org/10.1590/S008062342004000300003

Kyngäs, H. (2000). Compliance of adolescents with chronic disease. Journal of Clinical Nursing, 9, 549-556. https://doi.org/10.1046/j.1365-2702.2000.00368.x

Kyngäs, H., Kroll, T., \& Duffy, M. (2000). Compliance in adolescents with chronic diseases: A review. Journal of Adolescent Health, 26, 379-388. https://doi.org/10.1016/S1054-139X(99)00042-7

Lachman, M. (1986). Locus of control in aging research: A case for multidimensional and domain-specific assessment. Psychology and Aging, 1, 34-40. https://doi.org/10.1037/0882-7974.1.1.34

Lachman, M., Neupert, S., \& Agrigoroaei, S. (2011). The relevance of control beliefs for health and aging. In K. Schaie \& S. Willis (Eds.), Handbook of the psychology of aging ( $7^{\text {th }}$ ed., pp. 175-190). New York, NY: Elsevier.

Lao, R. (1974). The developmental trend of the locus of control. Personality and Social Psychology Bulletin, 1,348-350. https://doi.org/10.1177/0146167274001001117

Lazarus, R., \& Folkman, S. (1984). Stress, appraisal and coping. Nova York, NY: Springer.

Linhares, V., Meneses, R., Pais-Ribeiro, J. L., Pedro, L., Silva, I., Vilhena, E., et al. (2014). Preditores da adesão à medicação na epilepsia: Estudo longitudinal. Journal of Epilepsy and Clinical Neurophysiology, 20, 154-156. https://doi.org/10.15309/14psd150107

MacKinnon, D., \& Luecken, L. (2008). How and for whom? Mediation and moderation in health psychology. Health Psychology, 27, S99-S100. https://doi.org/10.1037/0278-6133.27.2(Suppl.).S99

Martin, L., Williams, S., Haskard, K., \& DiMatteo, M. (2005). The challenge of patient adherence. Therapeutics and Clinical Risk Management, 1(3), 189-199.

Mendonça, M., Andrade, C., \& Fontaine, A. M. (2009). Transição para a idade adulta e adultez emergente: Adaptação do Questionário de Marcadores da Adultez junto de jovens Portugueses. Psychologica, 51,147-168. https://doi.org/10.14195/1647-8606_51_10

Molassiotis, A., Nahas-Lopez, V., Chung, W., Lam, S., Li, C., \& Lau, T. (2002). Factors associated with adherence to antiretroviral medication in HIV-infected patients. International Journal of STD \& AIDS, 13, 301-310. https://doi.org/10.1258/0956462021925117

Nelson, M. C., Story, M., Larson, N. I., Neumark-Sztainer, D., \& Lytle, L. A. (2008). Emerging adulthood and college-aged youth: An overlooked age for weight-related behavior change. Obesity, 16, 2205-2211. 
https://doi.org/10.1038/oby.2008.365

Nunes, S., \& Tapadinhas, A. (2014). Crenças de saúde e locus de control no rastreio do cancro colo-rectal. In J. L. Pais-Ribeiro, I. Silva, R. Meneses \& I. Leal (Eds.), Atas do $10^{\circ}$ Congresso Nacional de Psicologia da Saúde (pp. 660-671). Porto: Sociedade Portuguesa de Psicologia da Saúde.

Pollack, M., Chastek, B., Williams, S. A., \& Moran, J. (2010). Impact of treatment complexity on adherence and glycemic control: An analysis of oral antidiabetic agents. Journal of Clinical Outcomes Management, 17(6), 257-265.

Reiners, A., Azevedo, R., Vieira, M., \& Arruda, A. (2008). Produção bibliográfica sobre adesão/não-adesão de pessoas ao tratamento de saúde. Ciência \& Saúde Coletiva, 13, 2299-2306. https://doi.org/10.1590/S1413-81232008000900034

Ridder, D., \& Schreurs, K. (2001). Developing interventions for chronically ill patients: Is coping a helpful concept. Clinical Psychology Review, 21, 205-240. https://doi.org/10.1016/S0272-7358(99)00046$\mathrm{X}$

Röder, I., \& Boekaerts, M. (1999). Stress, coping, and adjustment in children with a chronic disease: A review of the literature. Disability and Rehabilitation, 21, 311-337. https://doi.org/10.1080/096382899297576

Rodríguez-Rosero, J., Ferriani, M., \& Coleta, M. (2002). Escala de locus de controle da saúde- MHLC: Estudos de validação. Revista Latino-Americana de Enfermagem, $10,179-184$. https://doi.org/10.1590/S0104-11692002000200009

Rotter, J. (1966). Generalized expectancies for internal versus external control of reinforcement. Psychological Monographs: General and Applied, 80, 1-28. https://doi.org/10.1037/h0092976

Rotter, J. (1975). Some problems and misconceptions related to the construct of internal versus external control of reinforcement. Journal of Consulting and Clinical Psychology, 43, 56-67. https://doi.org/10.1037/h0076301

Rybarczyk, B., DeMarco, G., DeLaCruz, M., Lapidos, S., \& Fortner, B. (2001). A classroom mind/body wellness intervention for older adults with chronic illness: Comparing immediate and 1-year benefits. Behavioral Medicine, 27, 15-27. https://doi.org/10.1080/08964280109595768

Samson, A., \& Siam, H. (2008). Adapting to major chronic illness: A proposal for a comprehensive taskmodel approach. Patient Education and Counseling, 70, 426-429 https://doi.org/10.1016/j.pec.2007.10.018

Sawicki, G., \& Tiddens, H. (2012). Managing treatment complexity in cystic fibrosis: Challenges and opportunities. Pediatric Pulmonology, 47, 523-533. https://doi.org/10.1002/ppul.22546

Sawyer, S., Drew, S., Yeo, M., \& Britto, M. (2007). Adolescents with a chronic condition: Challenges living, challenges treating. Lancet, 369, 1481-1489. https://doi.org/10.1016/S0140-6736(07)60370-5

Sherbourne, C., Hays, R., Ordway, L., DiMatteo, M., \& Kravitz, R. (1992). Antecedents of an adherence to medical recommendations: Results from the medical outcomes study. Journal of Behavioral Medicine, 15, 447-468. https://doi.org/10.1007/BF00844941

Silver, E., Westbrook, L., \& Stein, R. (1998). Relationship of parental psychological distress to consequences of chronic health conditions in children. Journal of Pediatric Psychology, 23, 5-15. https://doi.org/10.1093/jpepsy/23.1.5

Sirey, J., Bruce, M., Alexopoulos, G., Perlick, D., Friedman, S., \& Meyers, B. (2001). Stigma as a barrier to recovery: Perceived stigma and patient-rated severity of illness as predictors of antidepressant drug adherence. Psychiatric Services, 52, 1615-1620. https://doi.org/10.1176/appi.ps.52.12.1615

Sousa, M., Landeiro, M., Pires, R., \& Santos, C. (2011). Coping e adesão ao regime terapêutico. Revista de Enfermagem Referência, 3(4), 151-160.

Storer, J., Cychosz, C., \& Anderson, D. (1997). Wellness behaviors, social identities and health promotion. American Journal of Health Behavior, 21(4), 260-268.

Strickland, B. (1989). Internal/external control expectancies: From contingency to creativity. American Psychologist, 44, 1-12. https://doi.org/10.1037/0003-066X.44.1.1

Suris, J., Michaud, P., \& Viner, R. (2004). The adolescent with a chronic condition. Part I: Developmental issues. Archives of Disease in Childhood, 89, 938-942. https://doi.org/10.1136/adc.2003.045369

Taddeo, D., Egedy, M., \& Frappier, J.-Y. (2008). Adherence to treatment in adolescents. Paediatrics \& Child Health, 13, 19-24. https://doi.org/10.1093/pch/13.1.19

Tillotson, L., \& Smith, M. (1996). Locus of control, social support, and adherence to the diabetes regimen. The Diabetes Educator, 22, 133-139. https://doi.org/10.1177/014572179602200206

Trevino, K., Fasciano, K., \& Prigerson, H. (2013). Patient-oncologist alliance, psychosocial well-being, and treatment adherence among young adults with advanced cancer. Journal of Clinical Oncology, 31, 1683-1689. https://doi.org/10.1200/JC0.2012.46.7993

Wall, L. (1992). The relationship between pain locus of control and treatment adherence at long-term 
follow-up from an interdisciplinary pain center. (Unpublished master's thesis). University of Nebraska Omaha, United States.

Wallston, K. (1982). Health locus of control beliefs. Patient Education Newsletter, 5, 56-57.

Wallston, K. (1992). Hocus-pocus, the focus isn't strictly on locus: Rotter's social learning theory modified for health. Cognitive Therapy and Research, 16, 183-199. https://doi.org/10.1007/BF01173488

Wallston, K. (2005). The validity of the Multidimensional Health Locus of Control scales. Journal of Health Psychology, 10, 623-631. https://doi.org/10.1177/1359105305055304

Wallston, K., Wallston, B., \& DeVellis, R. (1978). Development of the multidimensional health locus of control (MHLC) scales. Health Education Monographs, 6, 160-170. https://doi.org/10.1177/109019817800600107

Wiebe, J., \& Christensen, A. (1996). Patient adherence in chronic illness: Personality and coping in context. Journal of Personality, 64, 815-835. https://doi.org/10.1111/j.1467-6494.1996.tb00945.x

Williams, A., Manias, E., \& Walker, R. (2008). Interventions to improve medication adherence in people with multiple chronic conditions: A systematic review. Journal of Advanced Nursing, 63, 132-143. https://doi.org/10.1111/j.1365-2648.2008.04656.x

World Health Organization. (2010). ICD-10: International statistical classification of diseases and related health problems (4th ed). Geneve, Switzerland: Author.

World Medical Association. (2008). World medical association declaration of Helsinki: Ethical principles for medical research involving human subjects. Retirado de: http://www.wma.net/en/20activities/10ethics/10helsinki/

Wortman, C., \& Dunkel-Schetter, C. (1979). Interpersonal relationships and cancer: A theoretical analysis. Journal of Social Issues, 35, 120-155. https://doi.org/10.1111/j.1540-4560.1979.tb00792.x

Yeager, K., Dilorio, C., Shafer, P., McCarty, F., Letz, R., Henry, T., \& Schomer, D. (2005). The complexity of treatments for persons with epilepsy. Epilepsy \& Behavior, 7, 679-686. https://doi.org/10.1016/j.yebeh.2005.07.008

Historial do artigo

Recebido 02/08/2016

Aceite $\quad 24 / 07 / 2017$

Publicado $11 / 2017$

0 processo de revisão deste artigo foi responsabilidade da anterior direção da revista Psicologia 